\title{
STAT1 is involved in signal transduction in the EPO induced HEL cells
}

\author{
JIANG ChU*, ChANG Yun GUI*, JIE FAN**, \\ XIAO DA TANG**, Ruo LAN QIAO ${ }^{1}$ \\ * Shanghai Institute of Cell Biology, Chinese Academy of \\ Sciences, Shanghai 200031, China \\ ** Dept. of Urology, Shanghai First People's Hospital, \\ Shanghai 200081, China
}

\begin{abstract}
Erythropoietin (EPO) is the major regulator of mammalian erythropoisis, which stimulates the growth and differentiation of hematopoietic cells through interaction with its receptor (EPO-R). Here we use HEL cells (a human erythro-leukemia cell line) as a model to elucidate the pathway of signal transduction in the EPO-induced HEL cells. Our data show that the EPOR (EPO receptor) on the surface of HEL cells interacts with the Janus tyrosine protein kinase (Jak2) to transduce intracellular signals through phosphorylation of cytoplasmic proteins in EPO-treated HEL cells. Both STAT1 and STAT5 in this cell line are tyrosine-phosphorylated and translocated to nucleus following the binding of EPO to HEL cells. Furthermore, the binding of both STAT1 and STAT5 proteins to specific DNA elements (SIE and PIE elements) is revealed in an EPO-dependent manner. Our data demonstrate that the pathway of signal transduction following the binding of EPO to HEL cells is similar to immature erythroid cell from the spleen of mice infected with anemia strain of Friend virus.
\end{abstract}

Key words: Erythropoietin, HEL cells, signal transduction.

1. Corresponding author 
STAT1 involved in EPO- induced HEL cells

\section{INTRODUCTION}

The proliferation and differentiation of hematopoietic cells are regulated by growth factors or cytokines through binding to specific membrane receptors of their target cells[1]. Erythropoietin (EPO), a $34 \mathrm{kD}$ glycoprotein, is the principal growth factor regulating proliferation and differentiation of erythroid progenitors and exerts its action by the activation of receptors on the surface of these cells[2]. The genes encoding EPO and EPO receptor (EPOR) have been isolated, and the relationship between their structure and function has been extensively studied[3]. EPOR is a member of the superfamily of cytokine receptors that includes receptors for granulocyte colony-stimulating factor(G-CSF), granulocyte-macrophage colony-stimulating factor (GM-CSF), the interleukins (ILs) (IL-2, -3, -4, -5, -6, -7, -9, -11 and -12), growth hormone $(\mathrm{GH})$, and prolactin(PRL) $[4,5]$.

Activation of the EPOR triggers a cascade of phosphorylation/dephosphorylation reactions that are believed to be essential to EPO's signal transduction[6, 7]. Although the receptors of cytokines (such as EPO and IL3) lack intrinsic protein tyrosine kinase (PTK) activity, they can rapidly induce the phosphorylation of cytoplasmic proteins such as Jaks(Janus tyrosine protein kinases) and STATs. Jaks were found to play important roles in the transduction of intracellular signals[8]. Up to now, four Jaks (Jak1, 2, 3, and Tyk2) and eight STATs (STATl a, STAT1 $\beta$, STAT2, 3, 4, STAT5A, STAT5B and IL-4 STAT ) have been cloned and have been linked to the signal transduction pathways of individual cytokine[10].

Treatment of cells with IL-6, EGF (Epidermal Growth Factor) or PDGF (Platelet Derived Growth Factor) led to tyrosine phosphorylation of STAT1 or related proteins and the phosphorylated STAT1 can specifically bind to SIE (sis-inducible element or serum-inducible element)[11]. Previous studies demonstrated that EPO induced activation of STAT5 through Jak2 in progenitor cells and other cell lines such as BaF3, HCD57 cells, which were transfected with EPOR cDNA or infected by Friend Virus (FVA), respectively[12]. But little has been known about the signal transduction pathway in EPO-induced human erythroleukemia cells (such as HEL and K562 cells).

In this study we intend to reveal the signal transduction pathway in EPO-induced HEL cells, which possess endogenous EPOR, although previous studies have shown that there are only several decades of EPOR on the membrane of each HEL cell. These EPO receptors might be low affinity receptor, however, they still could respond to EPO stimulation. Our data demonstrate that EPO activates Jak2 and STAT5 in HEL cells. These results are much similar to those obtained from other cell lines and the erythroid progenitors[12]. In addition, our data reveal that EPO not only activates STAT5 but also activates STAT1 in EPO-induced HEL cells. Furthermore, the binding abilities of both STAT1 and STAT5 proteins to specific DNA elements are demonstrated. 


\section{MATERIALS AND METHODS}

\section{Materials}

Mouse monoclonal antibody of phosphotyrosine, the rabbit polyclonal antibodies (such as Jak1, Jak2, STAT1, STAT5), the related secondary antibodies (including HRP-conjugated sheep antirabbit and sheep anti-mouse immunoglobulin G) and protein A/G plus agarose beads were purchased from Santa Cruz Biotech. Protein molecular weight markers were purchased from Bio-Rad and Dalton company. The recombinant EPO was purchased from Kirin brewery company.

\section{Cell Culture}

HEL cells were maintained in RPMI-1640 medium (GIBCO-BRL) supplemented with $10 \%$ newborn calf serum, streptomycin $(100 \mu \mathrm{g} / \mathrm{ml})$, penicillin $(100 \mathrm{units} / \mathrm{ml})$ and glutamin $(2 \mathrm{mM})$. Before experiments HEL cells (control group and experimental group) were deprived of serum for $18 \mathrm{~h}$. For induced expeciments HEL cells were induced with EPO (10 units/ml) for $20 \mathrm{~min}$.

\section{The preparation of nuclear and cytoplasmic extracts}

Nuclear and cytoplasmic extracts were prepared as described previously[14, 15]. The protein concentration was determined according to Bradford's method.

\section{Immunoblotting (Western blot analysis)}

The nuclear and cytoplasmic extracts were prepared from EPO-treated and EPO-untreated HEL cells. The appropriate proteins were separated by SDS-PAGE and transferred to Hybond-C membranes (Amersham). The membranes were blocked in TBST buffer (10 $\mathrm{mM}$ Tris. HCl pH 7.6, $150 \mathrm{mM} \mathrm{NaCl}$ and $0.1 \%$ Tween-20) with 5\% nonfat milk (for regular Western blot assay) or with $1 \%$ BSA plus $1 \%$ nonfat milk (for detecting phosphotyrosine), then they were successively incubated with primary antibodies and HRP-labeled secondary antibodies for $2 \mathrm{~h}$. Finally the membranes were detected with ECL (Amersham).

\section{Immunoprecipitation with agarose conjugated antibodies}

$10 \mu \mathrm{l}(0.1 \mu \mathrm{g} / \mu \mathrm{l})$ of primary antibodies were added to $500 \mu \mathrm{l}$ of diluted nuclear or cytoplasmic extracts and incubated for $1 \mathrm{~h}$ at $4^{\circ} \mathrm{C}$, then $20 \mu \mathrm{l}$ of protein $\mathrm{A} / \mathrm{G}$ plus agarose beads were added and the mixture was rolled overnight at $4^{\circ} \mathrm{C}$. The beads were washed for 4 times with RIPA buffer (1 X Phosphorylated buffered solution, $1 \%$ NP 40, 0.5\% Sodium deoxycholate, $0.1 \%$ SDS, $0.1 \mathrm{mM}$ PMSF and $1 \mathrm{mM} \mathrm{Na} \mathrm{VO}_{4}$ ). Agarose beads were collected by microcentrifugation for 5 min at 4 ${ }^{\circ} \mathrm{C}$ and aspirated. $40 \mu \mathrm{l}$ of $1 \times$ electrophoresis sample buffer was added to the agarose beads and boiled for $3 \mathrm{~min}$. Finally, the immunoprecipitated proteins were separated on SDS-PAGE gels.

\section{The probe labeling}

The probes were labeled with $\gamma-{ }^{32}$ P-ATP for gel mobolity shift assay (GMSA) and Southwestern blot assay.

\section{The gel mobility shift assay (GMSA)[16]}

GMSAs were performed as previously described. The STAT1 a / $\beta$ specific binding sequence SIE (sis inducible element: 5'-GTG CAT TTC CCG TAA ATC TTG TCT ACA-3'), and the STAT5 specific binding sequence PIE (protactin inducible element: 5'-GGA CTT CTT GGA ATT AAG GGA-3') were used as probes. $8 \mu \mathrm{g}$ of nuclear extracts and $6 \mu \mathrm{g}$ of cytoplasmic proteins were incubated with or without unlabeled competitive oligonucleotides at $4^{\circ} \mathrm{C}$ for $2 \mathrm{~h}$. The positive control region (PCR) of human $\beta$-globin gene (5'-ACT GAT GGT ATG GGG CCA AGA GAT ATA TC-3') was used as a non-specific competitive oligonucleotide. 
STAT1 involved in EPO- induced HEL cells

\section{Southwestern blot assay[17]}

The nuclear extracts were separated by 10\% SDS-PAGE. The proteins were transferred from gel to membrane (Hybond-C) in the transfer buffer (glycine $39 \mathrm{mM}$, Tris base $48 \mathrm{mM}$, SDS $0.037 \%$, methanol 20\%) for $12 \mathrm{~h}$ at $4^{\circ} \mathrm{C}$. The membrane was blocked in Blotto (10 mM Tris. $\mathrm{HCl} \mathrm{pH} \mathrm{8.0,}$ $5 \%$ non-fat milk) for 1 hour at $4^{\circ} \mathrm{C}$, then was incubated with the labeled probe in the binding buffer (10 mM Tris $\mathrm{HCl} \mathrm{pH} \mathrm{8.0,} 50 \mathrm{mM} \mathrm{NaCl}, 10 \mathrm{mM} \mathrm{MgCl}_{2}, 0.1 \mathrm{mM}$ EDTA, $1 \mathrm{mM}$ DTT, 0.25\% non-fat milk) for $3 \mathrm{~h}$ at $4^{\circ} \mathrm{C}$. Finally washed with binding buffer and autoradiographed.

\section{RESULTS}

The nuclear and cytoplasmic proteins isolated from EPO-induced HEL cells were highly tyrosine-phosphorylated

Both nuclear and cytoplasmic proteins were prepared from EPO-induced and uninduced HEL cells and their phosphorylation was examined by using monoclonal antibody of phosphotyrosine. Our data showed that the nuclear and cytoplasmic proteins prepared from EPO-induced HEL cells were highly tyrosine phosphorylated (Fig 1, Lanes 1 and 3). However, tyrosine-phosphorylation in both nuclear and cytoplasmic proteins could not be detected in uninduced HEL cells (Fig 1, lanes 2 and 4). Furthermore, tyrosine-phosphorylated proteins in the cytoplasm of the EPOstimulated HEL cells were more than that in the nucleus of the same cells (Fig 1, lanes 3 and 1).

\section{MW(KD)}

Fig 1. Analysis of the tyrosine phosphorylation of cytoplasmic and nuclear extracts isolated from EPO-induced and uninduced HEL cells. The blot membrane was probed with mouse monoclonal antibody of phosphotyrosine.

Lane 1:40 $\mu \mathrm{g}$ of nuclear extracts from EPO-induced HEL cells.

Lane 2:40 $\mu \mathrm{g}$ of nuclear extracts from the uninduced HEL cells.

Lane 3: $40 \mu \mathrm{g}$ of cytoplasmic extracts from EPO-induced HEL cells.

Lane 4:40 $\mu \mathrm{g}$ of cytoplasmic extracts from the uninduced HEL cells.

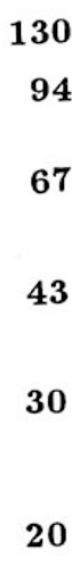

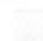


EPO-induced HEL cell, cytoplasmic extracts isolated from EPO-induced and uninduced HEL cells were immunoprecipitated with the agarose conjugated polyclonal antibodies of Jakl and Jak2. Our data showed that the tyrosine residues of Jak2 $(130 \mathrm{kD})$ were phosphorylated after HEL cells were induced with 10 units of EPO for $20 \mathrm{~min}$ (Fig 2A, Lane 2). However, Jak2 was not tyrosine-phosphorylated in the uninduced HEL cells (Fig 2A, Lane 1). In addition to Jak2, there were several tyrosine-phosphorylated proteins in the EPO induced HEL cells, suggesting that other cytoplasmic proteins might be activated by EPO (Fig 2A, Lane 2). On the other hand, the cytoplasmic proteins immunoprecipitated by Jakl antibodies did not show the $135 \mathrm{kD}$ protein band of tyrosine phosphorylation (Fig 2B, Lanes 1 and 2). Our data thus demonstrated that Jakl could not be activated in EPO-induced HEL cells. However, several other tyrosine-phosphorylated bands could be detected in the cytoplasmic extracts of EPO-induced HEL cells except Jakl (Fig 2B, lane 1). These results inferred that EPO stimulation might result in phosphorylation of

A

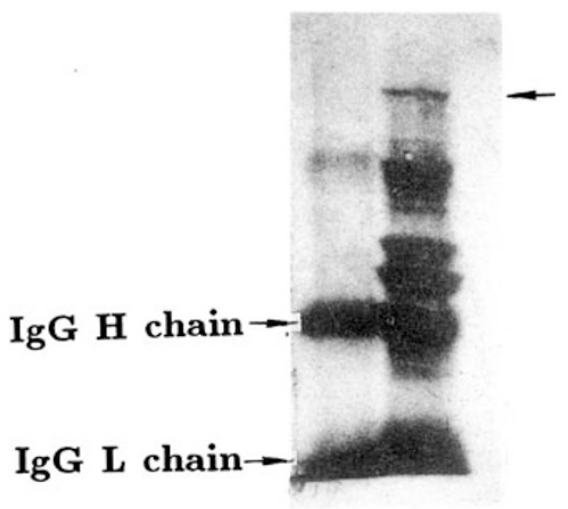

12
130

94

67

43

30

20

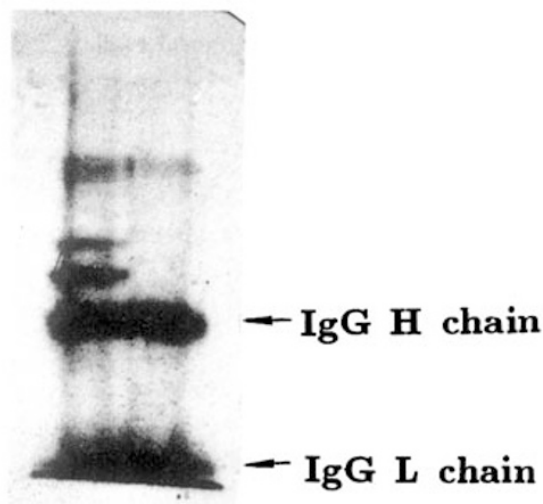

12

Fig 2. Analyses of the tyrosine phosphorylation of cytoplasmic extracts. The cytoplasmic proteins were immunoprecipitated with agarose-conjugated rabbit polyclonal antibodies of Jak2 (A) and Jak1 (B), respectively and then were examined with mouse monoclonal antibody of phosphotyrosine (pY). IgG $\mathrm{H}$ and $\mathrm{L}$ chains represent the positions of the heavy and light chains of rabbit polyclonal antibodies, respectively.

A: Lane 1: The cytoplasmic extracts from HEL cells deprived of serum.

Lane 2: The cytoplasmic extracts from induced HEL cells. The tyrosine phosphorylated Jak2 was marked with arrow (130KD).

B: Lane 1: The cytoplasmic extracts from induced HEL cells.

Lane 2: The cytoplasmic extracts from HEL cells deprived of serum. 
other proteins, which could bind to Jak1. All these results revealed that the effect of EPO on HEL cells was coupled with Jak2, not Jak1; and the activation of Jak2 was dependent on the interaction between EPO and EPOR in HEL cells.

\section{STAT 1 and STAT5 were tyrosine-phosphorylated and translocated to nucleus in EPO-induced HEL cells}

We observed that both STAT1 and STAT5 were tyrosine phosphorylated in the cytoplasm of HEL cells after these cells were induced by EPO (Fig 3A, Lane 2 and Fig 4A, Lane 1). Both STAT1 and STAT5 proteins were also detectable in the nucleus of EPO induced HEL cells (Fig 3B, Lane 2, 3 and Fig 4B, Lane 2). Other STAT proteins such as STAT3 could not be detected in the nucleus of induced HEL cells (data not shown). Our results showed that EPOR, Jak2, STAT proteins might be involved in response to the stimulation of EPO in HEL cells, and suggested that both STAT1 and STAT5 were tyrosine phosphorylated following the binding of EPO

$\mathbf{A}$

B

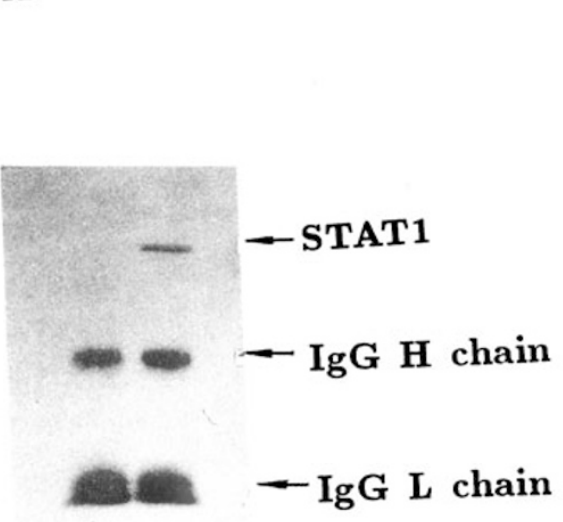

12

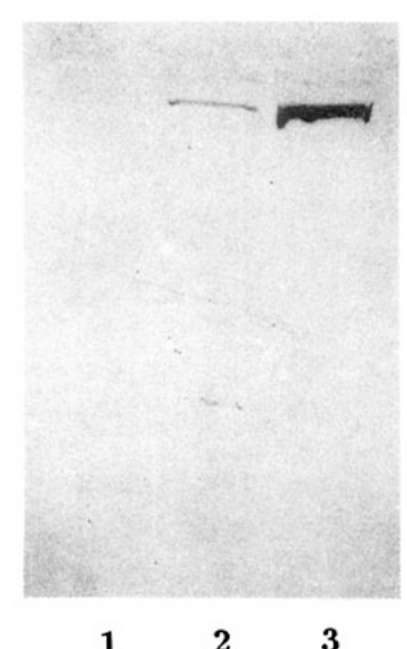

$\begin{array}{lll}1 & 2 & 3\end{array}$

Fig 3. Western blotting assay of STAT1 with anti-STAT1 polyclonal antibody.

A: The cytoplasmic proteins were immunoprecipitated by mouse monoclonal antibody of phosphotyrosine (pY) and then were probed with rabbit polyclonal antibody of STAT1.

Lane 1: $40 \mu \mathrm{g}$ of cytoplasmic extracts from HEL cells deprived of serum.

Lane 2: $40 \mu \mathrm{g}$ of cytoplasmic extracts from induced HEL cells. IgG H and L chains represent the migrating position of heavy and light chain of mouse IgG immunoglobin.

B: The nuclear extracts which were not immunoprecipitated by $\mathrm{pY}$ were probed with rabbit polyclonal antibody of STAT1.

Lane 1: $40 \mu \mathrm{g}$ of nuclear extracts from HEL cells deprived of serum. Lane 2: $40 \mu \mathrm{g}$ of nuclear extracts from HEL cells induced for $10 \mathrm{~min}$. Lane 3: $40 \mu \mathrm{g}$ of nuclear extracts from HEL cells induced for $20 \mathrm{~min}$. 


\begin{abstract}
A

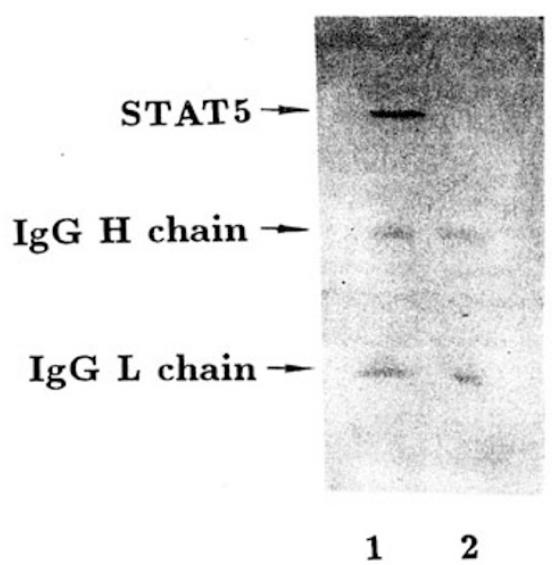

B

A: The cytoplasmic proteins were immunoprecipitated by mouse monoclonal antibody of phosphotyrosine (pY) and then were probed with rabbit polyclonal antibody of STAT5.

Lane 1: The cytoplasmic extracts from induced HEL cells.

Lane 2: The cytoplasmic extracts from HEL cells deprived of serum. IgG $\mathrm{H}$ and $\mathrm{L}$ chains represent the migrating position of heavy and light chain of mouse IgG immunoglobin.

B: The nuclear extracts which were not immunoprecipitated by $\mathrm{pY}$ were probed with rabbit polyclonal antibody of STAT5.

Lane 1: $40 \mu \mathrm{g}$ of nuclear extracts from HEL cells deprived of serum.

Lane 2: $40 \mu \mathrm{g}$ of nuclear extracts from induced HEL cells.
\end{abstract}

to HEL cells and then translocated to nucleus.

\title{
Characterization of EPO-dependent STAT1 and STAT5 DNA binding activity
}

Penta et al and Sawyer et al observed that EPO could activate STAT1 and STAT5 in immature erythroid cells prepared from the spleen of CD2F1 mice infected with the anemia-inducing strain of Friend Virus (FVA cells)[11, 18]. Furthermore, they demonstrated that STAT1 could bind to both PIE and SIE elements, STAT5 could only bind to PIE element. However, other workers showed that EPO could only activate STAT5 in the erythroid progenitors and leukemia cell line such as HCD57 cells[19]. To elucidate the mechanism of activation in EPO-induced HEL cells, gel mobility shift assays were performed by using SIE (the sis inducible element) and PIE (the prolactin inducible element) sequences as probes. When labeled SIE was used as a probe, no shift band was detected in the uninduced HEL cells (Fig 5, 
Lane 2). However one major shift band (band A) was revealed in EPO-induced HEL cells (Fig 5, Lane 3). Clearly, this band represents the binding of tyrosine phosphorylated STAT1 to SIE sequence, since in the competitive assay, band A could be competed by both PIE and SIE sequences (Fig 5, Lanes 4, 5 and 6) and it could not be competed by nonspecific oligonucleotide (PCR). However, band B could be competed by PCR (Fig 5, Lane 7), and therefore, this band might represent a nonspecific binding protein. When labeled PIE was used as probe, there was one shift band detected in the nuclear extracts of EPO-induced HEL cells (Fig 6, Lane 3 ) and no band could be detected in the nuclear extracts isolated from uninduced HEL cells (Fig 6, Lane 2). In the competitive assay, this band could completely be competed

Fig 5. Gel mobility shift assay for nuclear proteins with SIE consensus sequence as a probe. The nuclear proteins were isolated from EPO-induced and uninduced HEL cells. Unlabeled SIE, PIE and PCR oligonuceotides were used as competitors. Lane 1: Without proteins.

Lane 2: With $8 \mu \mathrm{g}$ nuclear proteins prepared from serum-deprived HEL cells.

Lane 3: With $8 \mu \mathrm{g}$ nuclear proteins prepared from induced HEL cells. Band A represents SIE-STAT1 complex.

Lane 4: Same as Lane 3, with 100-fold excess of unlabeled SIE oligonucleotide as a competitor.

Lane 5: Same as Lane 3, with 100-fold excess of unlabeled PIE oligonucleotide as a competitor.

Lane 6: Same as Lane 3, with 150-fold excess of unlabeled PIE oligonucleotide as a competitor.

Lane 7: Same as Lane 3, with 150-fold excess of unlabeled PCR oligonucleotide as a competitor. Band B might be nonspicific complex.

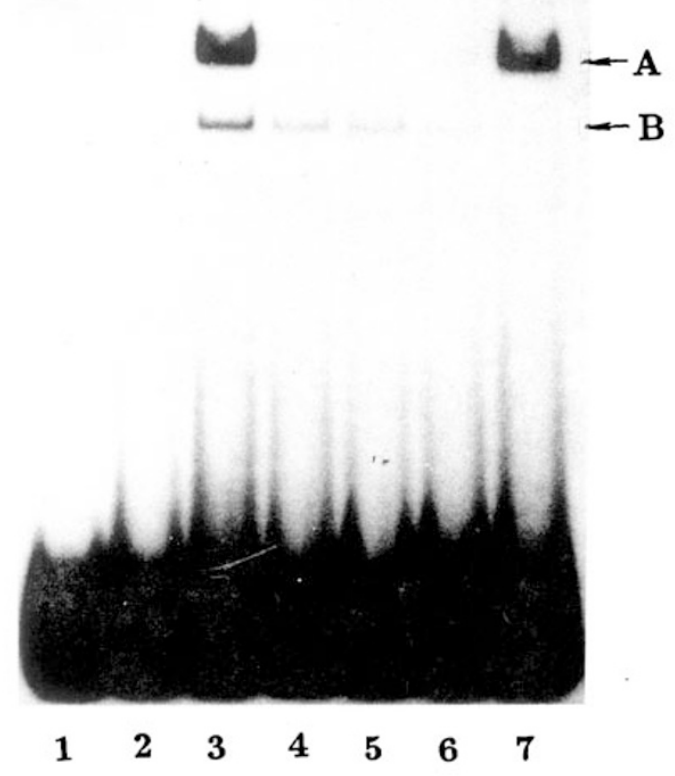

by PIE element (Fig 6, lane 4), but could not be competed by SIE element and nonspecific oligonucleotide PCR (Fig 6, Lane 5 and 6).

To further analyze the binding activities of STAT proteins, the cytoplasmic extracts were also examined by gel mobility shift assays. We found that there was only one band detected in EPO-induced HEL cells either by using SIE (Fig 7, Lane 3) or by using PIE as probes (Fig 8, Lane 3). The cytoplasmic extracts from the 
Fig 6. Gel mobility shift assay for nuclear proteins with PIE consensus sequence as a probe. The nuclear proteins were isolated from EPO-induced and uninduced HEL cells. Unlabeled PIE, SIE and PCR oligonuceotides were used as competitors. Lane 1: Without proteins.

Lane 2: With $8 \mu \mathrm{g}$ nuclear proteins prepared from serum-deprived HEL cells.

Lane 3: With $8 \mu \mathrm{g}$ nuclear proteins prepared from induced HEL cells. The band represents PIE-STAT5 complex.

Lane 4: Same as Lane 3, with 100-fold excess of unlabeled PIE oligonucleotide as a competitor.

Lane 5: Same as Lane 3, with 100-fold excess of unlabeled SIE oligonucleotide as a competitor.

Lane 6: Same as Lane 3, with 150-fold excess of unlabeled PCR oligonucleotide as a competitor.

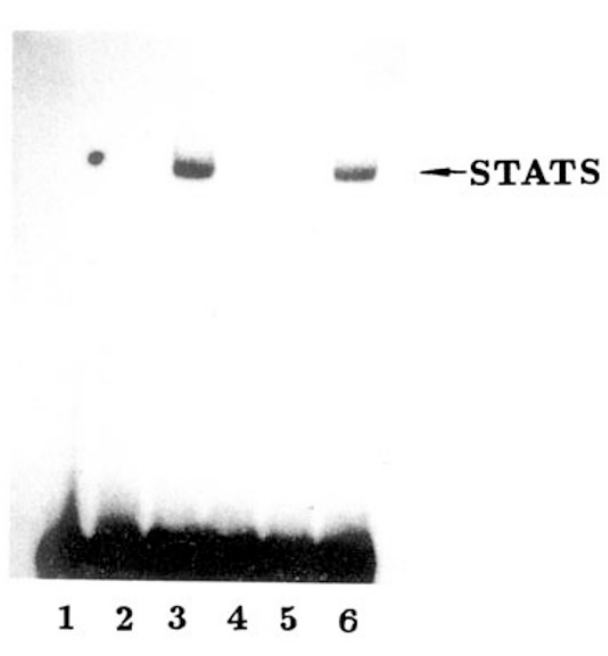

Fig 7. Gel mobility shift assay for cytoplasmic proteins with SIE consensus sequence as a probe. The cytoplasmic proteins were prepared from EPO-induced and uninduced HEL cells. Unlabeled SIE, PIE and PCR elements were used as competitors.

Lane 1: Without proteins.

Lane 2: With $6 \mu \mathrm{g}$ of cytoplasmic proteins prepared from HEL cells deprived of serum.

Lane 3: With $6 \mu \mathrm{g}$ of cytoplasmic proteins prepared from induced HEL cells. The band represents SIESTAT1 complex.

Lane 4: Same as Lane 3, with 100-fold excess of unlabeled SIE oligonucleotide as a competitor.

Lane 5: Same as Lane 3, with 150-fold excess of unlabeled PIE oligonucleotide as a competitor.

Lane 6: Same as Lane 3, with 150-fold excess of unlabeled PCR oligonucleotide as a competitor.

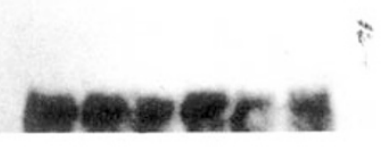

$\begin{array}{llllll}1 & 2 & 3 & 4 & 5 & 6\end{array}$ 
control HEL cells deprived of serum could not form any complex with SIE or PIE probes (Fig 7, Lane 2 and Fig 8, Lane 2). Using SIE element as a probe, the band could be competed by both SIE and PIE (Fig 7, Lanes 4 and 5). But could not be competed by PCR (Fig 7, Lane 6). Using PIE element as a probe, the band could be competed by unlabeled PIE element, (Fig 8, Lanes 4 and 6), but could not be competed by unlabeled SIE (Fig 8, Lane 5). These results further confirm that STAT1 can bind to both PIE and SIE elements, since STAT5 can only bind to PIE element, but not to SIE sequence.

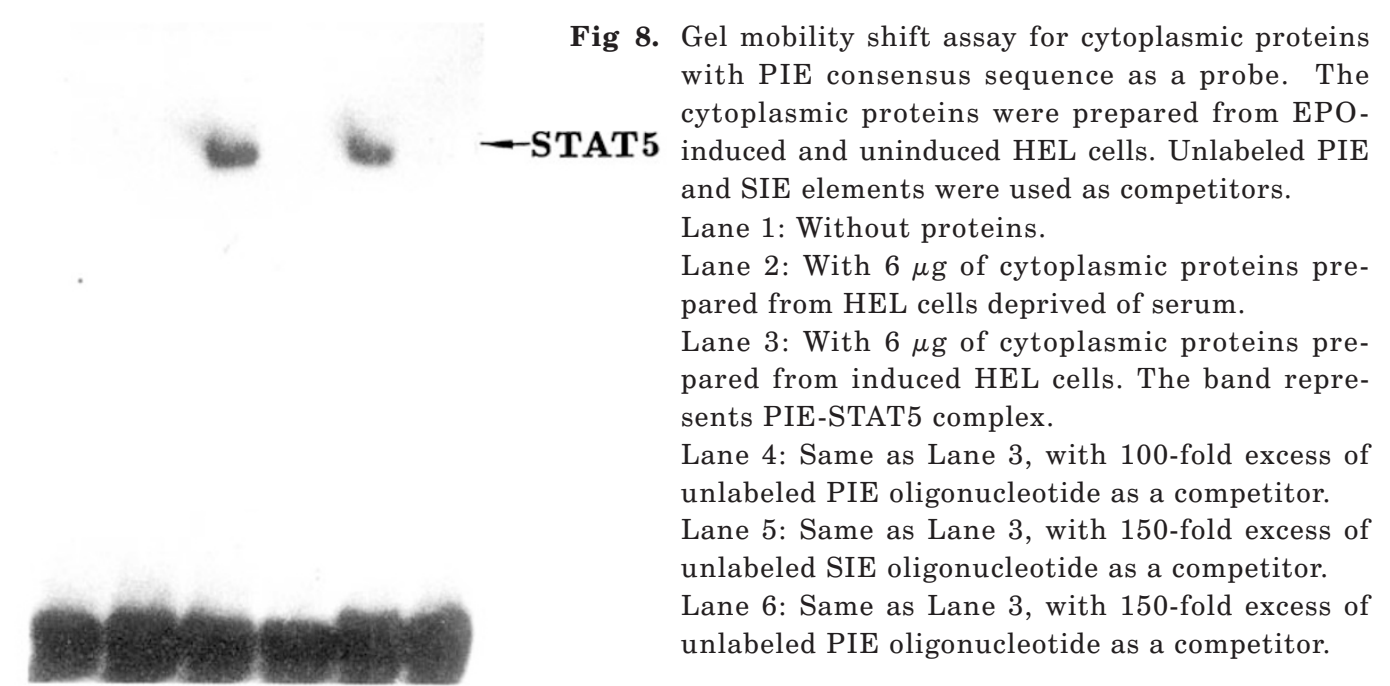

$\begin{array}{llllll}1 & 2 & 3 & 4 & 5 & 6\end{array}$

Taken together, all these results revealed that tyrosine-phosphorylation is necessary for the activation of both STAT1 and STAT5 binding abilities.

The SIE and PIE binding proteins were tyrosine-phosphorylated STAT1 and STAT5

To confirm the molecular weight of DNA binding proteins in the gel mobility shift assays, Southwestern blot assays were performed. No band could be detected by using labeled SIE element as a probe with the nuclear extracts of uninduced HEL cells (Fig 9, Lane 1). However, with nuclear extacts from EPO-induced HEL cells, one specific band could be detected which represented tyrosine-phosphorylated STAT1 protein (including STAT1 a and STAT1 $\beta$ ) based on its molecular weights 
(84/91 kD, Fig 9, lane 2). Similarly, by using labeled PIE element as a probe, the $95 \mathrm{kD}$ phosphorylated STAT5 binding protein was obvious (Fig 10, Lane 3). These results were consistent with the result of competitive GMSA, and both STAT5 and STAT1 were activated in EPO-treated HEL cells. With respect to other bands in Fig 10, we suggested that they might represent fragments spliced from STAT5 or some unknown proteins activated by EPO and could weakly bind to PIE probe. Our results also demonstrated that the PIE binding activities following EPO induction for 20 minutes were stronger than that for 10 minutes EPO-induction (Fig 10, Lanes 2 and 3$)$.

\section{MW(KD)}

130

Fig 9. Southwestern blotting assay: ${ }^{32}$ p-labeled SIE element is used as a probe. The band represents the complex formed by the SIE probe with the tyrosine-phosphorylated $\operatorname{STATl}(\alpha+\beta)$.

Lane 1: With $80 \mu \mathrm{g}$ of nuclear proteins prepared from the uninduced HEL cells. No band was formed.

Lane 2: With $80 \mu \mathrm{g}$ of nuclear proteins prepared from the induced HEL cells.

\author{
67 \\ 43 \\ 30
}

94

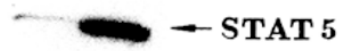

20

\section{$\operatorname{MW}(\mathbf{K D})$}

130

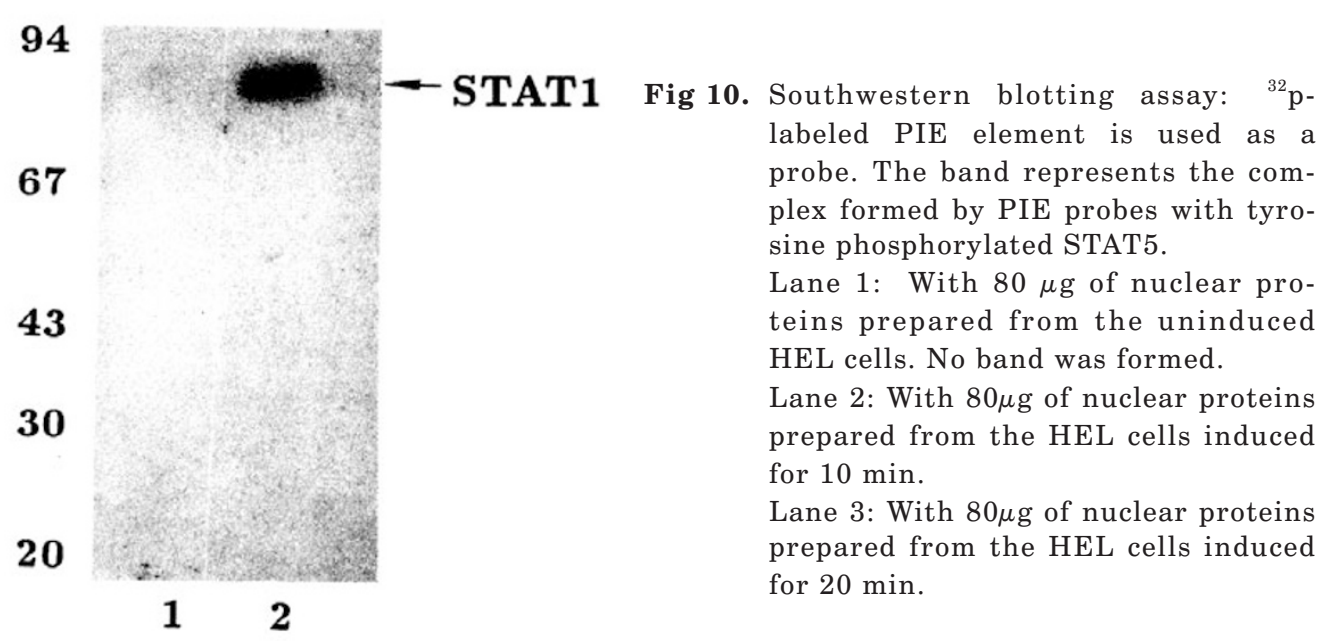


STAT1 involved in EPO- induced HEL cells

\section{DISCUSSION}

Miruo et al (1994) demonstrated that Janus tyrosine protein kinases (Jak2) might be associated with the EPO receptor following the binding of EPO. With HEL cells (a human erythroleukemia cell line) as a model to elucidate the molecular mechanism involved in the induction of EPO, the present data demonstrated that both cytoplasmic and nuclear proteins were highly tyrosine-phosphorylated in EPO induced HEL cells and Jak2, but not Jak1, was tyrosine-phosphorylated. Moreover, it is probable that phosphorylated tyrosine of EPO receptor could bind to Jak2. However, Jak1 might be associated with some tyrosine-phosphorylated cytoplasmic proteins that were coprecipitated by antibody of Jak1. This is why we can detect several tyrosine phosphorylated proteins in EPO-induced HEL cytoplasm by using the antibody of Jak1 (Fig 2 B, Lane 1). In addition, the cytoplasmic proteins such as STAT1 and STAT5 are also tyrosine-phosphorylated following the Jak2 activation.

Previous studies showed that STAT1 and STAT5 could be activated in the immature erythroid progenitors prepared from spleen of CD2F1 mice infected with Friend Virus following the induction of $\operatorname{EPO}[11,18]$. However, other studies with erythroid and non-erythroid cells transfected with EPOR cDNA just showed that STAT5 was tyrosine phosphorylated by the stimulation of EPO[19]. Our results firmly demonstrated that EPO could activate STAT1 as well as STAT5 in EPO-induced HEL cells, in which tyrosine residues were phosphorylated and the activated STAT proteins were translocated from cytoplasm to nucleus, whereby displaying their binding abilities to specific SIE and PIE elements respectively. Interestingly, the activated STAT1 and STAT5 could exist in both cytoplasmic and nuclear extracts at the same time. So, it seems that the activation and translocation might be proceeded at the same time.

In order to test the presence of activated STAT1 protein in the nuclear extract of EPO-induced HEL cells, GMSA was performed by using labeled SIE as a probe. The results indicated that there was a band of specific STAT1-SIE complex (band A, Fig 5, Lane 3) which could be completely competed by both SIE and PIE elements, but not by non-specific PCR oligonucleotides. However, in the same figure, there was a weaker band (band B) which could be competed partially by SIE and PIE elements and completely by PCR oligonucleotides. This result is difficult to explain, but we have the opinion that it may be due to the presence an unknown nuclear protein which could directly or indirectly bind to multiple elements.

\section{REFERENCES}

[1] Schindler c JE, Darnell Jr. Transcriptional Responses to polypeptide ligands: the Jak-STAT Pathway. Ann Rev Biochem 1995; 64:621-51.

[2] Youssoufion Hagop, Gregory Longmore, Drorit Neumann, Ailihiko Yochimura, Harvey F Lodish. Structure, Function and Activation of the Erythropoietin Receptor. Blood 1993; 81:2223-36.

[3] Jones S Sirnon, Alan D D'andrea, Lora L Haines, Gordon G Wong. Human Erythropoietin 
Receptor: Cloning, Expression and Biologic characterization. Blood 1990; 76:31-35.

[4] D'Andrea AD, Fasman GD, Lodish HF. A new hematopoietic growth factor receptor superfamily. Curr Opin Cell Biol 1990; 2:648.

[5] D'Andrea AD, Fasman GD, Lodish HF. Erythropoietin receptor and interleukin 2 receptor Beta chain: A new receptor family. Cell 1990; $58: 1023$.

[6] Wakao Hiroshi, Noboyuk Harada, Toshio Kifamura, ALF Mui, Alsushi Miyajima. Interleukin 2 and erythropoitin activate STATS/MGF via distinct pathways. EMBO J 1995; 14:2527-35.

[7] Klingmuller Usula, Svethana Bergelson, Janathan G Hsiao, Harrey F Lodish. Multiple tyrosine residues in the cytosolic domain of the erythropoietin receptor promote activation of STAT 5. Proc Natl Acad Sci USA 1996; 93:8324-8.

[8] Bittorf Thomas, Robort Jaster, Britta Luotke, Beutrice Kamper and Josef Brok. Requirement of Jak 2 in erythropoietin-induced signaling pathway. Cell Signal 1997; 9:85-9.

[9] Ihle JN, Witthuhn BA Quelle FW, Yamamoto K, Thierfelder WE, Kreider B, Silvennoinen O. Signaling by the cytokine receptor superfamily Jaks and STATs. Trends Bio chem Sci 1994; 19:222.

[10] Darnell JE, Jr Kerr, IM Stark GR. Jak-STAT pathways and transcriptional activation in response to IFNs and other extracellular signaling proteins. Science 1994; 264:1415.

[11] Penta K, ST Sawyer. Erythropoietin induces the tyrosine phosphorylation, Nuclear translocation and DNA binding of STAT1 and STAT5 in erythroid cells. J Biol Chem 1995; 270:31282-87.

[12] Pallard C, F Gouillenx, M Charon, B Groner, S Gisselbrecht, I Dusanter Fourt. Interleukin-3, Erythropoietin and prolactin activate a STAT5-1ike factor in lymphoid cells. J Biol Chem 1995; 270:15942-5.

[13] Gouilleux Fabrice, Caroline Pallard, Isabelle, Dusanter-Fuort Hiroshi Wakao, Lars-Arne Haldosen, Grunnar Norstedt, David Levy and Bernd Groner. Prolactin, growth hormone, erythropoietin and granulocyte-macrophage colony stimulating factor induce MGF-STAT5 DNA binding activity. EMBO J 1995; 14:2005-13.

[14] Gorski KM, Carneiro U. Schibler. Tissue-specific in vitro transcription from the mouse albumin promoter. Cell 1986; 47:767-76.

[15] Ausubel FM, Brent R, Kingston RE, Moore DD, Seidman JG, Smith JA, Struhl K. Short protocols in Molecular Biology. 2th edition.

[16] Patel HR, AJ Sytkowski. Erythropoietin activation of AP1. Exp Hemat 1995; 23:619-25.

[17] Bading Hilmar. Determination of the molecular weight of DNA-bound protein(s) responsible for gel electrophoretic mobility shift of linear DNA fragment exemplified with purified viral myb protein. Nucleic Acids Research 1988; 16:5241-8.

[18] Ohashi Takashi, Michiaki Masuda, Sundra Ruscetti. Induction of sequence-specific DNAbinding factors by erythropoietin and the spleen focus-forming virus. Blood 1995; 85:1454-62.

[19] Jacobs-Helber MS, K Penta, Z Sun, A Lawson, ST Sawyer. Distinct signaling from stem cell factor and erythropoietin in HCD57 cells. J Biol Chem 1997; 272:6850-3.

Received March-3-1998. Revised May-18-1998. $\quad$ AcceptedMay-5-1998. 